\title{
DeWiz - Modular Debugging for Supercomputers and Computational Grids
}

\author{
Dieter Kranzlmüller \\ GUP Linz, Johannes Kepler University Linz, \\ Altenbergerstr. 69, A-4040 Linz, Austria/Europe, \\ kranzlmueller@gup.uni-linz.ac.at, \\ http://www.gup.uni-linz.ac.at/
}

\begin{abstract}
Debugging is accepted as one of the difficult tasks of high performance software development, which can be attributed to the high complexity of parallel and distributed applications. Especially users of massively parallel supercomputers or distributed metacomputer systems experience huge obstacles, that are difficult if not impossible to overcome with existing error detection approaches. The prototype tool DeWiz presents an effort to improve this situation by applying the abstract event graph model as a representation of parallel program behavior. Besides its usability for different programming paradigms it permits analysis of data with various debugging activities like automatic error detection and sophisticated abstraction. In addition, DeWiz is implemented as a set of loosely connected modules, that can be assembled according to the user's needs and given priorities. Yet, it is not intended as a complete replacement but as a plug-in for well-established, existing tools, which may utilize it to increase their debugging functionality.
\end{abstract}

\section{Introduction}

The quest for ever increased computational performance seems to be a neverending story mainly driven by so-called "grand challenge" problems of science and engineering, like simulations of complex systems such as weather and climate, fluid dynamics, and biological, chemical, and nuclear reactions. Since existing computing systems allow only insufficient calculations and restricted solutions in terms of processing speed, required memory size, and achieved numerical precision, new architectures and approaches are being developed to shift the performance barrier. The upper limit of this development is represented by supercomputer systems, which may be further coupled to metacomputers. Recent examples of realization projects are the US Accelerated Strategic Computing Initiative (ASCI) [6] and the multi-institutional Globus project [4. While ASCI seeks to enable Teraflop computing systems far beyond the current level of provided performance, the Globus project tries to enable computational grids that provide pervasive, dependable, and consistent access to distributed highperformance computational resources.

Such systems achieve their level of performance due to their high degree of powerful parallel and distributed computing components. Yet, this complicates 
the software development task due to the required coordination of multiple, concurrently executing and communicating processes. As a consequence, big obstacles are experienced during all phases of the software lifecycle, which initiated many research efforts to improve the parallel programmers situation with manifold strategies and development tools.

One area of investigation is testing and debugging, which shares a great part in determining the reliability of the application and thus the quality of the software. The goal of debugging is to detect faulty behavior and incorrect results occurring during program execution, which is attempted by analyzing a program run and investigating process states and state changes. Obviously, the complexity of the program and the number of interesting state changes determines the amount of work needed to analyze the program's behavior. This means, that bigger systems are probably more difficult to debug than smaller systems. Yet, there are only a limited number of parallel debuggers, that are suitable for error detection of applications running on massively parallel and distributed systems. The biggest problem is that current debugging tools suffer from managing the amount of presented data, which stems from mainly two characteristics: Firstly, most parallel debugging tools are composed from combing several sequential tools and integrating them under a common user interface. These tools often lack support for detecting errors derived from parallelism. Secondly, many tools are based on textual representations, which may be inappropriate in many cases to display and manage the inherent complexity of parallel programs 13 .

The work described in this paper differs from existing approaches due to the fact, that debugging activities are based on the event graph model instead of the underlying source code. It describes a parallel program's execution by occurring state changes and their interactions on concurrently executing processes, which allows to cope equally with programs based on message-passing and the shared memory paradigm. Furthermore, it can be applied for automatic error detection and to perform higher-level program abstraction.

These ideas are implemented in the tool prototype DeWiz, the Debugging Wizard. In contrast to other tools, DeWiz does not contain a user interface for the analysis task, but instead offers its results to other existing debugging tools. By providing an adaptable input interface, traces from post-mortem debuggers and event-streams from on-line debuggers can be processed. Similarly, an output interface allows to use DeWiz as a plug-in for the user's preferred analysis tool. Another feature is its steering module, that offers a way for the user to describe the desired analysis more precisely. This covers the idea, that the user has knowledge about the program's expected behavior and may thus be able to identify different priorities for more or less critical errors and analysis tasks.

This paper is organized as follows. The next section discusses the target systems for our debugging model and the requirements imposed onto parallel debugging tools. Afterwards, the event graph model is introduced and some possibilities for analyzing a program's behavior are presented. This leads to the 
actual implementation of DeWiz in Section 4 , which is described by its main features and its mode of operation.

\section{Requirements to a Parallel Debugger}

The main criterion for any tool developer is a definition of target systems, which in our case are high performance supercomputers. Since there exist different architectures and possibilities of programming them, a wide variety of strategies and tools have already been proposed. In the case of parallel debuggers many promising approaches exist as academic or research prototypes, for example Mantis [1], P2D2 [5], PDBG [2], and PDT 1], or even as a commercial tool like Totalview [3. A characteristic of these systems is, that every approach applies several instances of an existing sequential debugger in order to perform the debugging task on the participating processes. Although this may be useful in most cases, it introduces some obstacles especially on large scale computing systems like massively parallel machines or heterogenous clusters of supercomputers.

The problems experienced during debugging of supercomputer applications are mostly connected to the amount of data that has to be analyzed. Firstly, these programs tend to be long-lasting, from some hours to several days or even more. As a consequence, many state changes occur that have to be observed and processed by the debugging tool. In the worst case, debugging a supercomputer may require another supercomputer to perform the analysis task. Secondly, the execution time and the large number of participating processes leads to enormous interprocess relations, which cannot be comprehended by the user. Thirdly, a great amount of debugging activities has to be performed equally for different processes and repeated iterations of the target application.

Previous solutions were always based on down-scaling, which means that the program's size is reduced in terms of participating processes and executed numbers of iterations. While this may be successful in many cases, it also contains potential for critical errors, which may be experienced only in full-scale real-world applications. As a consequence, there may be some cases where the program has to be tested under regular conditions in its intended environment. In order to comply to this requirement, we have identified the following characteristics for a debugger of the above mentioned target systems:

- Heterogeneity: supporting different programming models and architectures.

- Usability: managing huge amounts of data and improving program understanding.

- Abstraction: reducing the amount of debugging data presented to the user.

- Automatization: performing repeated debugging activities without user interaction.

A strategy or tool supporting these characteristics may then be applicable to full-scale applications, and may allow to perform debugging activities impossible with existing solutions. In addition, it may also improve the error detection task on smaller-scale application sizes, especially if it can be combined with other tools in this area. 


\section{The Event Graph Model for Debugging}

Our solution to fulfil the characteristics described above are based on the event graph model. An event graph is a directed graph $G=(E, \rightarrow)$, where $E$ is the nonempty set of vertices $e$ of $G$, while $\rightarrow$ is a relation connecting vertices, such that $x \rightarrow y$ means that there is an edge from vertex $x$ to vertex $y$ in $G$. The vertices $e$ are the events occurring during program execution, which change the state of the corresponding process 14. The relation establishes Lamport's "happened before" ordering [10, which consists of the sequential order on one particular process and the order between disjunct processes whenever communication or synchronization takes place.

In principle, every change of process state is caused by an event, and there are huge numbers of events being generated during program execution. However, usually only a small subset of events is required during debugging, which allows to filter only interesting events for error detection and to reduce the number of state changes for investigation. The remaining events are then collected as the vertices of the event graph. One difficulty is to define, which events to collect and which state changes to ignore. In order to allow a large degree of flexibility, the events collected in the event graph are user-defined. For example, a user looking for communication errors may define point-to-point communication events as established by send and receive function calls to be the target of investigation.

During program analysis, it is not only important to know about the occurrence of an event, but also about its properties. These properties are called event attributes and represent everything that may be interesting for the investigator. Similar to the events, the set of attributes may be appointed by the user. For the above mentioned communication points, a user may identify the communication statement's parameters to be event attributes for the analysis. Another kind of attributes are source code pointers, which consist of filename and line number corresponding to the original function call or statement. These attributes are needed in order to establish a connection between the graph and the faulty source code.

With the event graph model defined as above it is possible to describe erroneous behavior. In principle, every error theory defines two groups of bugs in programs, failures and computational errors. While the former is clearly recognizable, e.g. through program break-downs or exceptions being taken, the latter always depends on the semantic contents of the results and requires a verification step. Thus, computational errors can only be detected by comparison of expected results with actually obtained results.

Integrating failure detection in the event graph model is relatively easy, since their occurrence usually leads to an end of the corresponding process. Thus, a failure is always the last event on a particular process, which is characterized by having only one approaching edge but no leaving edge. Therefore, a debugging tool can easily direct the programmer's attention to such places by analyzing the final event on each participating process.

On the other hand, computational errors may occur at both, edges and vertices of the event graph. Since the edges describe a set of state changes, and these 


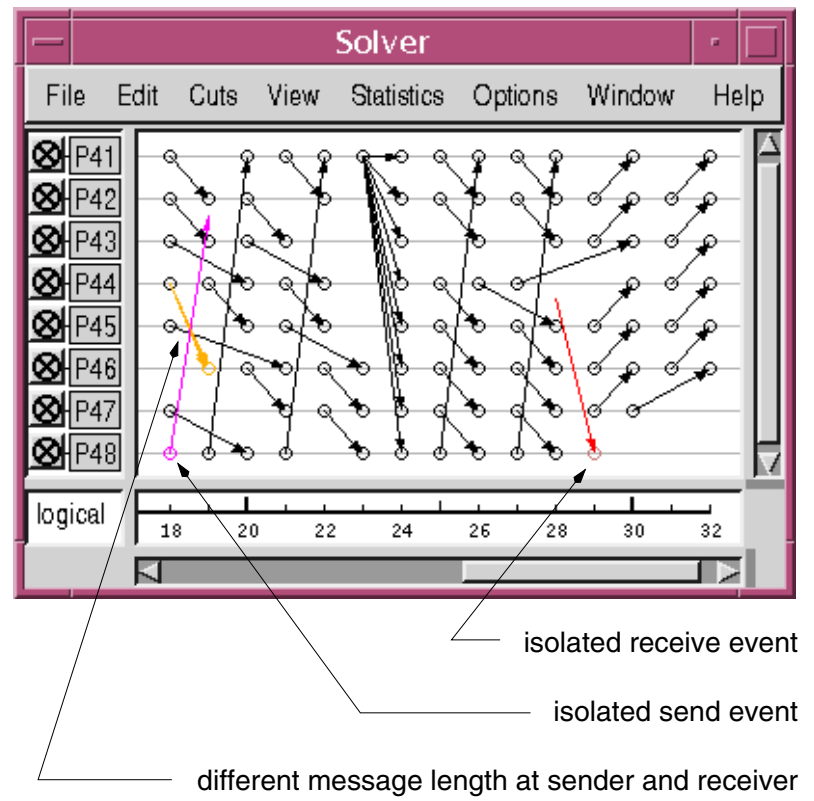

Fig. 1. Basic event graph analysis (e.g. errors in point-to-point communication)

state changes determine the results of the program, analyzing the edges may be required in order to detect incorrect behavior. Errors at vertices can be identified by describing the expected event attributes. If the event attributes obtained during execution differ from the expected data, incorrect or illegal operations have been detected. Please note, that incorrect operations may not necessarily result in errors, e.g. when the program is prepared to handle such unexpected events.

For instance, comparing expected and actual attributes of the communication events may expose isolated events or events with different message length. Isolated events are send events without corresponding receive events or vice versa. Events with different message length are revealed, if the size of the message data differs at sender and receiver. However, even if events with these characteristics are detected, they need not necessarily result in malign behavior. For example, isolated send events may have no effect, while isolated receive events may block the processes' execution forever.

An example of these basic debugging features is visible in Figure 1 It shows the execution of a program on 8 selected nodes from a possibly much larger execution. Some of the edges in the graph are highlighted to emphasize errors in the communication parameters of corresponding send and receive function calls.

Besides the basic analysis capabilities of checking event attributes, a more sophisticated way of analysis considers the shape of the graph itself. Often, a set of corresponding send and receive events resembles more or less complex 


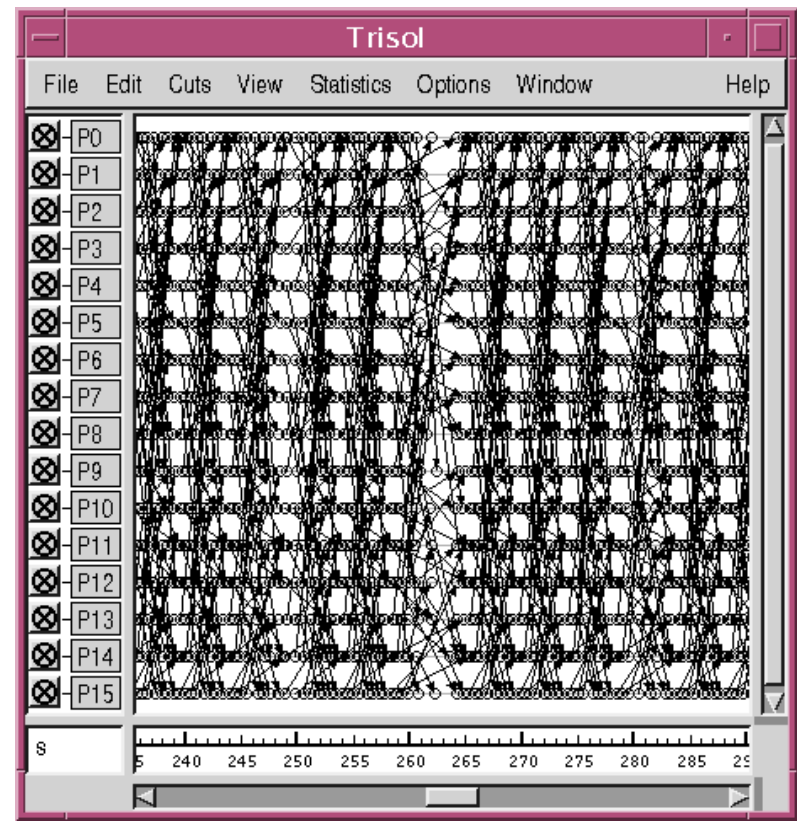

Fig. 2. Advanced event graph analysis (e.g. anomalous event graph pattern)

communication patterns, like broadcast, scatter, gather, and butterfly. Other possibilities are iterations in the observed algorithm, repeated function calls to selected communication statements, and grouping of processes (see 7] for an overview of some examples). These characteristics can be detected with simple pattern matching algorithms. As a result, this analysis allows to detect complete patterns, nearly complete patterns, and the absence of expected patterns in the event graph.

An example for communication pattern analysis is visible in Figure 2, This shows a finite element solver that was executed on 16 nodes. There have been 200 iterations to perform the computation, and only point-to-point communication events have been traced. In total, the trace contained around 20.000 events. Therefore, it is possible that the strange behavior during one of the operations could have remained unnoticed, especially if a smaller scale than seconds would have been selected. With pattern matching, this strange behavior would have been detected immediately. Please note, that this is a real example and we detected this error only by accident, before we developed this strategy.

The next step after detecting anomalous behavior is to direct the users attention to such places in the event graph. This is called automatic abstraction and means, that only a limited surrounding of the erroneous events is extracted. Therefore, instead of presenting all the data to the user, only critical sections of the event graph are displayed. A simplified operation of automatic abstraction 
is to evaluate the history of events that finally resulted in the detected bug. Therefore, it may only be necessary to display the communication partners of the corresponding process, and this only for a certain interval of time. As a consequence, in contrast to displaying huge numbers of events and several hundred processes, only a small subset is presented to the user, which still contains the interesting places for the debugging task.

\section{DeWiz, the Debugging Wizard Prototype Tool}

The event graph model as described above together with a preliminary set of error detection functions has been implemented in a tool prototype called DeWiz. Besides this functionality, several aspects have been applied during the design of DeWiz, which are as follows:

- Modularity: the debugging functionality must be adaptable to the users needs and the applications characteristics.

- Independence: the features of DeWiz must be applicable without a defined graphical user interface in mind, but instead as a plug-in to available debugging tools.

- Efficiency: due to the prospected amount of data, the tool must be implemented in order to facilitate all available performance by executing modules in parallel and applying parallelized algorithms during the analysis (e.g. pattern matching).

In addition, DeWiz contains a steering module, that allows to integrate the users knowledge into the debugging task. Within the proposed strategy it is able to allow the users to define

- events interesting for monitoring,

- expected behavior in terms of communication patterns, and

- priority between different kinds of errors

These aspects are available in a first tool prototype, whose operation during program analysis is displayed in Figure 3. The starting point is a target system, that is observed by an available monitoring tool. This monitoring tool is connected to DeWiz either on-line via event streams or post-mortem via tracefiles. In order to combine the tool with an available monitoring utility, the input interface has been adapted to process the given event data. This input interface forwards the data to the modules of DeWiz, which perform the desired analysis.

At this point, the users knowledge interferes with the systems operation. A dedicated steering module allows to decide about the expected behavior and the priority of different errors. The user with the knowledge about the target system, called the debugging expert, enters this data via a configuration file. In the future, we will change this propriety form of steering with some kind of graphical user interface. The configuration given by the user determines the 


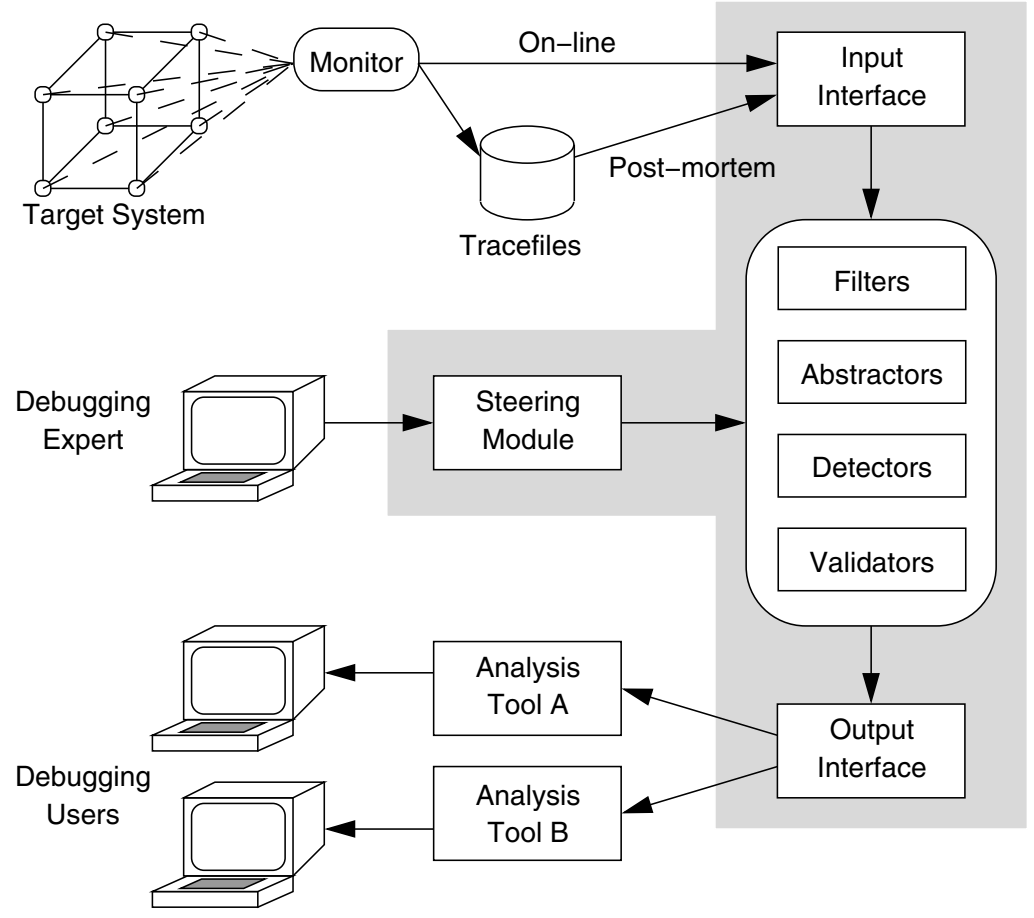

Fig. 3. Flowdiagram of DeWiz during opertation

arrangement of the working modules. Clearly, modules evaluating higher priority errors are executed earlier during the analysis task. In addition, the distribution of the modules to available processing elements can be defined in order to improve the analysis speed.

After the analysis has be completed, or critical errors have been detected, the results are forwarded to the output interface. Again, at this end of the tool, some customizations have to be carried out to provide the results of DeWiz to other existing analysis tools. Of course, since the results are delivered as event streams, any possible connection as well as concurrent connections to different tools are imaginable.

At present, the current prototype of DeWiz has been connected to the MAD environment [8], which has been developed at our department. Besides others, MAD contains an event monitoring utility that performs the program observation of MPI programs 11], and several analysis tools that visualize the evaluated analysis data. One of these analysis tools is ATEMPT, which visualizes a statetime diagram of the programs execution. The results of DeWiz are therefore mapped onto the display of ATEMPT, and allow to remove all analysis functionality from the graphical user interface. In addition, it is much easier to extend 
the modular approach of DeWiz, and as a side effect provide these extensions to other available debugging environments.

\section{Conclusions and Future Work}

The work described in this paper tries to provide a solution for the difficulties encountered during debugging of massively parallel supercomputers as well as distributed metacomputers. With the event graph model it is relatively easy to distinguish correct and incorrect behavior, to provide some means of abstraction and to perform tedious analysis tasks automatically.

The presented ideas have already been implemented in the DeWiz tool prototype, which has been successfully connected to an existing debugging environment. In addition, the modular composition of DeWiz allows to easily extend and improve its functionality, which is one of the current goals in this project. By providing more analysis features, the capabilities of DeWiz can be increased with benefits for the debugging user. It is even imaginable to develop a component library of parallel programming debugging modules, that can arbitrarily arranged by the users.

Another aspect experienced during our research is, that many analysis tasks are really time-consuming, especially with the large amounts of data to be processed. Thus, it seems necessary to further optimize and parallelize some of the existing modules in order to speed up the execution.

A near future goal of this project is the extension of the input and output interfaces. So far, we have only processed post-mortem tracefiles, and one of the next goals is to integrate an interface based on the OMIS monitoring standard [16]. This will allow us more flexibility in choosing the input connection and processing different event streams, and will probably deliver useful feedback for additional analysis modules.

Acknowledgements. This work represents an extension of my $\mathrm{PhD}$ thesis [9] and covers aspects that have not been described before. Consequently, many of the ideas presented here evolved from the many discussions with my PhD supervisor, Prof. Dr. Jens Volkert.

\section{References}

1. C. Clemencon, J. Fritscher, R. Rühl, "Visualization, Execution Control and Replay of Massively Parallel Programs within Annai's Debugging Tool", Proc. High Performance Computing Symposium, HPCS'95, Montreal, Canada, pp. 393-404 (July 1995).

2. J. C. Cunha, J. Lourenço, J. Vieira, B. Moscão, and D. Pereira., "A framework to support parallel and distributed debugging", Proc. of HPCN'98, High Performance Computing and Networking Europe, Amsterdam, Netherlands, (1998).

3. Etnus (Dolphin Interconnect Solutions Inc.): TotalView 4.1.0, Documentation: http: //www . etnus. com/pub/totalview/tv4.1.0/totalview-4.1.0-doc-pdf.tar, Framingham, Massachusetts, USA, 2000.

4. I. Foster, C. Kesselman, "The Globus Project: A Status Report", Proc. IPPS/SPDP'98 Heterogenous Computing Workshop, pp. 4-18 (1998). 
5. R. Hood, "The p2d2 Project: Building a Portable Distributed Debugger", Proc. SPDT'96, ACM SIGMETRICS Symposium on Parallel and Distributed Tools, Philadelphia, USA, pp. 127-136 (May 1996).

6. F. Hossfeld, "Teraflops Computing: A Challenge to Parallel Numerics?" Proc. 4th Intl. ACPC Conference, Springer, LNCS, Vol. 1557, Salzburg, Austria, pp. 1-12 (Feb. 1999).

7. D. Kranzlmüller, S. Grabner, J. Volkert, "Event Graph Visualization for Debugging Large Applications", Proc. SPDT'96, ACM SIGMETRICS Symposium on Parallel and Distributed Tools, Philadelphia, PA, pp. 108-117 (May 1996).

8. Kranzlmüller, D., Grabner, S., Volkert, J., Debugging with the MAD Environment, Parallel Computing, Vol. 23, No. 1-2, pp. 199-217 (Apr. 1997).

9. Kranzlmüller, D., Event Graph Analysis for Debugging Massively Parallel Programs, PhD Thesis, GUP Linz, Joh. Kepler University Linz,

http://www.gup.uni-linz.ac.at/ dk/thesis, (Sept. 2000).

10. Lamport, L., Time, Clocks, and the Ordering of Events in a Distributed System, Communications of the ACM, pp. 558 - 565 (July 1978).

11. S.S. Lumetta, D.E. Culler, "The Mantis Parallel Debugger", Proc. of SPDT'96: SIGMETRICS Symposium on Parallel and Distributed Tools, Philadelphia, PA, pp. 118-126 (May 1996).

12. Message Passing Interface Forum, "MPI: A Message-Passing Interface Standard Version 1.1", http://www.mcs.anl.gov/mpi/ (June 1995).

13. C.M. Pancake, "Visualization Techniques for Parallel Debugging and PerformanceTuning Tools", in: A.Y.Zomaya, "Parallel Computing: Paradigms and Applications", Intl. Thomson Computer Press, pp. 376-393 (1996).

14. M. van Rick, B. Tourancheau, "The Design of the General Parallel Monitoring System", Programming Environments for Parallel Computing, IFIP, North Holland, pp. 127-137 (1992).

15. M. Stitt, "Debugging: Creative Techniques and Tools for Software Repair", John Wiley \& Sons, Inc., NY (1992).

16. R. Wismüller, "On-Line Monitoring Support in PVM and MPI", Proc. EuroPVM/MPI'98, LNCS, Springer, Vol. 1497, Liverpool, UK, pp. 312-319, (Sept 1998). 